\title{
Early intervention for adolescents with borderline personality disorder using cognitive analytic therapy: randomised controlled trial
}

Andrew M. Chanen, Henry J. Jackson, Louise K. McCutcheon, Martina Jovev, Paul Dudgeon, Hok Pan Yuen, Dominic Germano, Helen Nistico, Emma McDougall, Caroline Weinstein, Verity Clarkson and Patrick D. McGorry

\section{Background}

No accepted intervention exists for borderline personality disorder presenting in adolescence.

\section{Aims}

To compare the effectiveness of up to 24 sessions of cognitive analytic therapy (CAT) or manualised good clinical care (GCC) in addition to a comprehensive service model of care.

\section{Method}

In a randomised controlled trial, CAT and GCC were compared in out-patients aged 15-18 years who fulfilled two to nine of the DSM-IV criteria for borderline personality disorder. We predicted that, compared with the GCC group, the CAT group would show greater reductions in psychopathology and parasuicidal behaviour and greater improvement in global functioning over 24 months

\section{Results}

Eighty-six patients were randomised and 78 (CAT $n=41$; GCC $n=37$ ) provided follow-up data. There was no significant difference between the outcomes of the treatment groups at 24 months on the pre-chosen measures but there was some evidence that patients allocated to CAT improved more rapidly. No adverse effect was shown with either treatment.

\section{Conclusions}

Both CAT and GCC are effective in reducing externalising psychopathology in teenagers with sub-syndromal or fullsyndrome bipolar personality disorder. Larger studies are required to determine the specific value of CAT in this population.

\section{Declaration of interest}

None. Funding detailed in Acknowledgements.
Borderline personality disorder is a severe mental disorder that usually emerges during adolescence, ${ }^{1}$ and adolescents with this disorder commonly seek clinical help. ${ }^{2,3}$ We have previously reviewed the prospects for developing prevention and early intervention strategies for the disorder ${ }^{4}$ and concluded that current evidence supports indicated prevention, ${ }^{5}$ targeting groups with precursor signs and symptoms such as substance use disorders or borderline personality disorder traits, ${ }^{6}$ along with early intervention for first presentations of borderline personality disorder.

We report a hybrid efficacy/effectiveness ${ }^{7}$ randomised controlled trial of early intervention for adolescents with sub-syndromal or full-syndrome borderline personality disorder using a novel, time-limited psychotherapy, cognitive analytic therapy, ${ }^{8}$ compared with 'manualised', structured, team-based, non-specialised 'good clinical care' specifically developed for this trial. Based upon our review, ${ }^{4}$ we predicted that, compared with good clinical care, cognitive analytic therapy would perform significantly better in decreasing borderline psychopathology, general psychopathology (internalising and externalising psychopathology) and parasuicidal behaviours (suicide attempts and non-suicidal self-injury) ${ }^{9}$ and in improving social and occupational functioning.

\section{Method}

\section{Participants}

Participants were aged 15-18 years, were sufficiently fluent in English and fulfilled two to nine DSM-IV criteria for borderline personality disorder. ${ }^{1}$ Participants were also required to have had one or more of the following in childhood: any personality disorder symptom, any disruptive behaviour disorder symptom, low socio-economic status, depressive symptoms and a history of abuse or neglect. These factors were selected because of their high odds ratios for the development of personality disorders in young adults $^{10,11}$ and because they were measurable in our sample.

Exclusion criteria were learning disability, psychiatric disorder due to a general medical condition, pervasive developmental disorder, severe primary Axis I disorder that should be the principal focus of treatment (e.g. medically unstable anorexia nervosa or severe obsessive-compulsive disorder) and receiving more than nine sessions of specialist mental health treatment in the previous 12 months. No potential participant was excluded on the basis of these criteria. Potential participants were not approached if they had sustained psychosis and met criteria for ORYGEN Youth Health's Early Psychosis Prevention and Intervention Centre. ${ }^{12}$

\section{Procedure}

The study was approved by the North Western Health Care Network Behavioural and Psychiatric Research and Ethics Committees. It was conducted from October 2000 to October 2004 at the Helping Young People Early (HYPE) clinic, a specialised early intervention programme for borderline personality disorder at ORYGEN, ${ }^{12}$ the government-funded mental health service for young people aged 15-18 years in western metropolitan Melbourne, Australia. Referrals to ORYGEN are taken directly from the community (via emergency departments, primary care, family, school or self-referral) for acute, severe mental health problems. The referrals are not specifically for borderline personality disorder treatment.

After complete explanation of the study procedures, written informed consent was obtained from all participants and their parent or guardian where appropriate. Eligibility criteria were first assessed by a full clinical interview, supplemented by the 
Structured Clinical Interview for DSM-IV Axis I Disorders Patient Version (SCID-I/P) ${ }^{13}$ the Schedule for Affective Disorders and Schizophrenia for School-Age Children - Present and Lifetime version (K-SADS-PL) disruptive behaviour disorders module, ${ }^{14}$ and the Structured Clinical Interview for DSM-IV Axis II Disorders (SCID-II) borderline personality disorder module. ${ }^{15}$ Final diagnosis was by a consensus group, comprising at least two senior investigators (A.C., H.J., L.M., P.M.), using a modified longitudinal, expert, all data (LEAD) standard. ${ }^{16}$ Participants were remunerated with AU\$20 for the first three research assessments and AU $\$ 40$ for the final follow-up to cover out-of-pocket expenses.

Participants were allocated to cognitive analytic therapy (CAT) or good clinical care (GCC) using a stratified block randomisation procedure with block size equal to four. This was designed and overseen by a statistician (H.P.Y.) who was masked to patient data. Stratification was according to the number of DSM-IV borderline personality disorder criteria (cut-off point 5). In order to accommodate the requirements of the clinical service, randomisation occurred following informed consent but prior to baseline assessment. The nature of the randomisation procedure was concealed from the therapists by using an on-site, password-protected computer program, operated by an independent ORYGEN administrative staff member who entered all participants' data. The administrative staff member communicated the outcome of the randomisation to the therapist and the therapist discussed this with the patient.

\section{Research assessments}

Assessments were conducted independently by three graduate research assistants (V.C., C.W., E.M.) trained by the principal investigator (A.C.) and supervised by another senior investigator (H.J.) who was masked to patient names and treatment allocation. No 'unmaskings' were reported during the conduct of the trial. Research assistant masking was tested upon completion of their employment (without warning) by asking them to guess each participant's treatment allocation using a checklist. Kappa values were $0.36,0.07$ and 0.06 respectively, indicating satisfactory masking.

Figure 1 shows the flow of participants through the trial. Participants were assessed at four fixed time points: baseline $(n=78), 6$ months $(n=70), 12$ months $(n=70)$ and 24 months ( $n=68)$. Assessments were completed for at least three of the four time points in $92 \%$ of the sample.

\section{Diagnostic interviews}

Diagnoses were obtained using the SCID-I/P, the K-SADS-PL disruptive behaviour disorders module and the full SCID-II. In keeping with previous research, a personality disorder criterion was scored positive if it had been present for 2 years and did not occur exclusively during an Axis I disorder. ${ }^{17}$ Also, antisocial personality disorder criterion A (age 18 years) was ignored and 'personality disorder not otherwise specified' was defined as either nine positive personality disorder criteria across any personality disorder domains or falling one criterion short of 'caseness' for a specific personality disorder diagnosis but having two additional criteria from any other personality disorder domain. ${ }^{2}$

\section{Outcome measures}

Primary outcome variables were defined a priori. We anticipated that the CAT group would show a greater reduction in psychopathology and parasuicidal behaviour (suicide attempts and

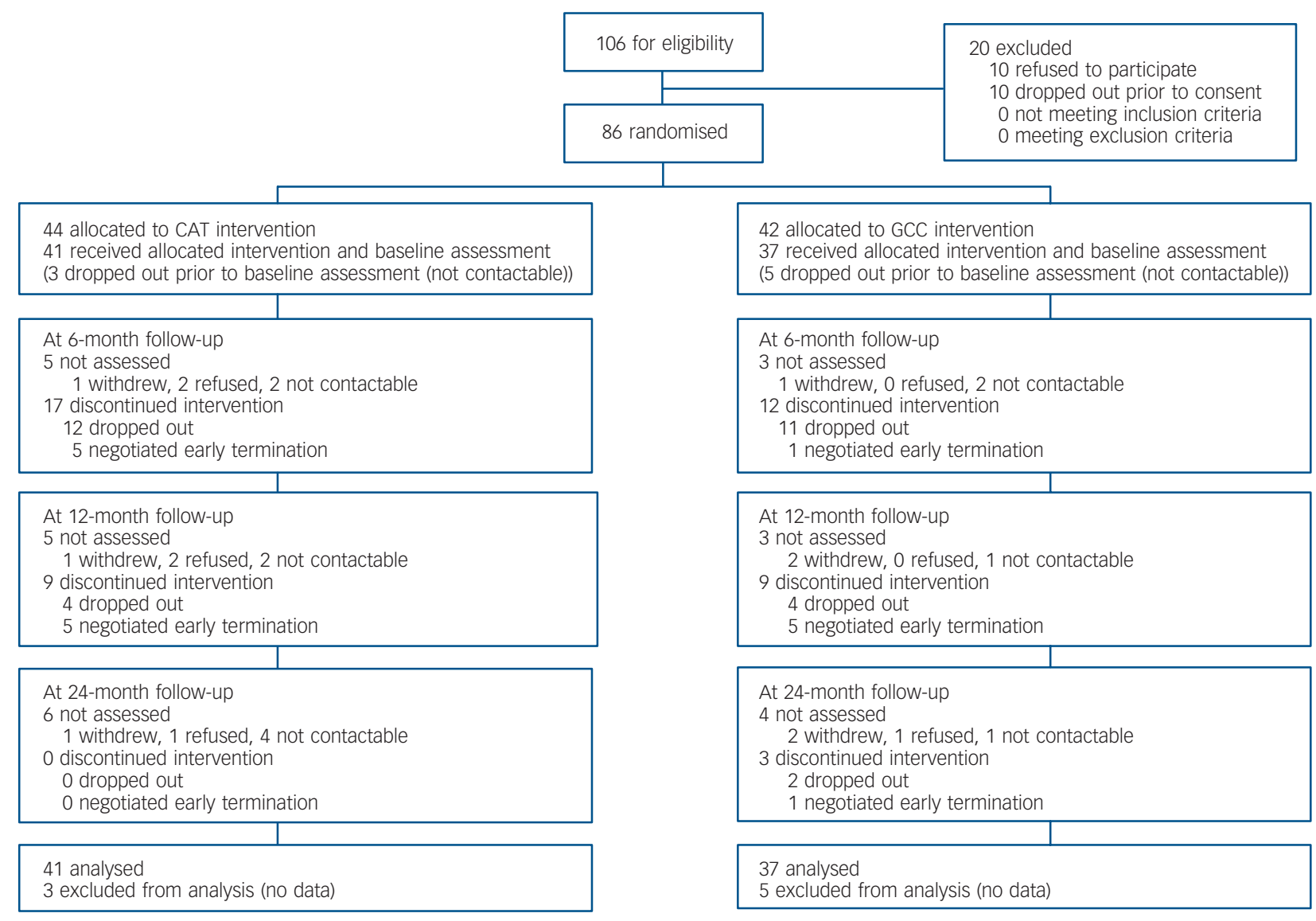

Fig. 1 Trial profile (CAT, cognitive analytic therapy; GCC, good clinical care). 
non-suicidal self-injury $)^{9}$ and greater improvement in global functioning.

\section{Psychopathology}

The SCID-II borderline personality disorder dimensional score was derived by summing the nine SCID-II items, scored 1 (absent), 2 (sub-threshold) or 3 (present). Scores range from 9 to 27. For follow-up assessments, each borderline personality disorder criterion was rated for the interval between assessments.

Internalising and externalising psychopathology scores were derived from the Youth Self-Report (YSR) questionnaire, a widely used instrument which assesses behavioural and emotional functioning in young people aged 11-18 years. ${ }^{18,19}$ It has 112 items, rated 0 (not true), 1 (somewhat or sometimes true) and 2 (very true or often true). The Young Adult Self-Report (YASR) is an analogue of the YSR for people aged 18-30 years, containing 116 items rated using the same YSR response format. ${ }^{20}$ As some of the items of the internalising and externalising sub-scales differ between the YSR and YASR, in keeping with previous research we calculated the mean item scores for each scale to ensure comparability. ${ }^{2}$ The resulting scores ranged between 0 and 2 .

\section{Parasuicidal behaviour}

Parasuicidal behaviour was assessed by semi-structured interview (developed by the investigators and available upon request) that included enquiring into each form of parasuicide and the number of episodes. The number of episodes was then coded as none, monthly, weekly or daily.

\section{Global functioning}

Global functioning was assessed using the widely used Social and Occupational Functioning Assessment Scale (SOFAS). ${ }^{21}$

\section{Treatment conditions and therapists}

Both forms of treatment comprised up to 24 weekly sessions.

\section{Therapists}

The three therapists (two female, one male) were 6th-year degree clinical psychologists (standard for Australia), trained in cognitive-behavioural therapy, with at least 2 years post-training experience. In order to control for therapist effects such as gender, training and experience, they delivered both interventions and the accompanying case management.

\section{Cognitive analytic therapy}

Cognitive analytic therapy is a time-limited, integrative psychotherapy developed in the UK over the past 25 years by Ryle $\&$ Kerr. $^{22}$ This therapy arose from a theoretical and practical integration of elements of psychoanalytic object relations theory and cognitive psychology, developing into an integrated model of development and psychopathology. It has increasingly been used with complex and relational disorders, especially borderline personality disorder. ${ }^{8,23}$ We selected this therapy because it had been developed for use in public health services and was suitable for early intervention. Drs Anthony Ryle and Ian Kerr conducted initial training over 9 months, comprising $100 \mathrm{~h}$ of face-to-face, large- and small-group seminars in Melbourne and London, and telephone-supervised practice. Study recruitment commenced when Dr Ryle judged that all three therapists were adherent to the therapy.
Each case in this arm of the study was supervised by an expert from the Association for Cognitive Analytic Therapy in the UK. Dr Ryle conducted a weekly telephone supervision group for some cases; all other cases were individually supervised, using weekly emailed process notes and monthly telephone calls. At the end of each session, the therapist summarised the session with the patient. The recording of this summary was converted to a secure mp3 file and emailed with the process notes. The summaries and notes were rated for treatment integrity and used for supervisor feedback.

\section{Standardised good clinical care}

Standardised good clinical care was a modular treatment package developed specifically for this study (further information available from the authors) and explicitly designed for the same purpose as Linehan's 'treatment by experts, ${ }^{24}$ namely to control for some factors commonly believed to be effective in psychotherapy (availability, accessibility and duration of therapy, institutional prestige and general factors associated with receiving therapy). This intervention was designed to deliver standardised, high-quality, team-based clinical care that might be achievable in mental health services in more economically developed countries. It used a simple problem-solving model for all participants, ${ }^{25}$ with additional modules determined by the co-occurring problems (e.g. depression, anxiety or anger management) identified by the patient and/or therapist. Some modules included basic cognitivebehavioural concepts such as identifying that thoughts and feelings are related and the use of monitoring and challenging unhelpful thoughts or beliefs. Supervision occurred weekly in a peer group led by a senior clinical psychologist external to the study.

\section{Common elements of the treatment models}

Both treatments used common elements of the HYPE service model of care and had equal access to assertive case management, psychiatrist appointments, activity groups, crisis team and in-patient care and pharmacotherapy (as indicated). As the principal investigator (A.C.) was also the study psychiatrist, all decisions to initiate pharmacotherapy were reviewed and approved by an experienced psychiatrist independent of the study.

\section{Treatment integrity}

In addition to the use of treatment manuals, expert training and supervision, all sessions were audio-recorded. All therapists and some supervisors audited randomly selected whole-session recordings to provide qualitative feedback to therapists contemporaneous with the trial. Cognitive analytic therapy was rated for adherence and competency by supervisor monitoring of the use of the specific 'tools' of the therapy (narrative formulation, diagrammatic formulation and 'goodbye letter') at the appropriate phase of therapy and by using the Therapist Intervention Checklist (Bennett, personal communication, 2006), short version rating of the session summary recording and detailed process notes. Four domains of this checklist were rated on a scale of 1-4 (1, well done; 2 , partially done; 3 , inadequately or not done; 4 , hammering or 'overkill'):

(a) noting key contents of the session

(b) using contents to propose links and explore possible patterns

(c) inviting patient's reaction to the therapy/session

(d) proposing/elaborating procedural descriptions of these reactions.

Total scores ranged from 4 to 16 , where scores below 8 indicated satisfactory adherence. Cognitive analytic therapy 
supervisor ratings for 163 therapy sessions across 25 patienttherapist dyads had a mean score of 6.3 (s.d.=1.1).

Treatment adherence for good clinical care and differentiation of this treatment from cognitive analytic therapy used a 21-item scale devised by C.W. and L.M. (further information available from the authors). Items drawn from the respective treatment manuals were rated as either present or absent, and included 10 prescribed items corresponding to the GCC sub-scale (scoring range $0-10)$ and 11 proscribed items corresponding to the CAT sub-scale (scoring range $0-11$ ).

Thirty-seven recordings were selected randomly from early, middle or late therapy (sessions 3, 12 or 20) and rated across 37 patient-therapist dyads (by C.W.). Mean score for the GCC subscale was 8.83 (s.d. $=0.25$ ), indicating excellent adherence. Mean score for the CAT sub-scale was 0.52 (s.d. $=0.11$ ), indicating negligible 'contamination' of good clinical care with elements of cognitive analytic therapy.

\section{Treatment of missing data and statistical methods}

Sample size was determined using the SCID-II borderline personality disorder dimensional score. A magnitude of three points difference (i.e. one borderline personality disorder feature) between the two treatment groups was considered clinically important. Past research data from our programme gave us a standard deviation of 3.6 for this score, producing an expected effect size of 0.8 (3/3.6). Therefore, we sought to detect a medium to large effect size (i.e. $0.6-0.8$ ) and with alpha set at 0.05 and power at 0.8 , a sample size of $26-45$ in each group would be needed. ${ }^{26}$ We therefore aimed to have a sample of 40 in each group.

Data analysis was by intention to treat. Missing values for the CAT and GCC groups on outcome measures due to withdrawal or non-attendance subsequent to baseline were assumed to have occurred at random, conditional on the completely observed pre-treatment scores on all outcome measures (see, for example, Schafer \& Graham, ${ }^{27}$ for a general review of missing data and their treatment). Ten multiply imputed data-sets were generated using the PAN package in the $\mathrm{R}$ statistical program, ${ }^{28,29}$ for which the imputation model was the same as the one used in the actual statistical modelling of the data.

Treatment differences and change over times were analysed by using the xtmixed procedure in Stata version 9.2 for Windows for the continuous total borderline personality disorder, SOFAS, internalising and externalising outcome measures and by using the gllamm package for the ordinal categorical parasuicidal behaviour. The four time points were coded as $-1,-0.75$, -0.5 and 0 in all models, thereby implying that regression coefficients involving time measured the linear rate of change from baseline to 24-month follow-up and that regression intercepts referenced group differences at the last follow-up point (no evidence of non-linear change in either the CAT or the GCC group was observed or found in preliminary models).

A linear random intercept model best fitted the SOFAS and internalising measures, whereas total borderline personality disorder and externalising outcomes were best represented by a linear random intercepts and slopes model. Parasuicidal behaviour was best fitted by a logistic proportional odds random intercepts model. Effects for all outcome measures were adjusted by additionally incorporating into all fitted models time-varying covariates for total SCID-II antisocial personality disorder score, presence of a mood disorder, and presence of a substance use disorder.

Only primary model parameters directly relevant to the study objectives are presented here. They are: first, group differences at
24 months (indicating whether cognitive analytic therapy was better or worse than good clinical care at the final follow-up); second, the linear rate of change from baseline to final followup for the GCC group (indicating the extent to which the reference group improved or deteriorated over the 24 months of the study); and third, differential rate of change for the CAT group (indicating whether the rate of improvement or deterioration in this group was substantially stronger than in the GCC group). All model parameters for continuous outcome measures are presented here as partial standardised effects, whereas those for the categorical measures of parasuicidal behaviour are presented as conditional odds ratios. Complete tables of all modelling results are available upon request from the authors.

The ten multiply imputed data-sets provided ten sets of model parameter estimates and their standard errors. These values were averaged over all ten imputed data-sets using Rubin's rules for scalar estimands to obtain mean multiply imputed model parameter estimates. ${ }^{30}$ Finally, 95\% confidence intervals were calculated for all parameter estimates using Rubin's Student's $t$ approximation. ${ }^{30}$

\section{Results}

The characteristics of participants ( $n=41$ CAT, $n=37$ GCC) are shown in online Table DS1.

\section{Treatment received}

The flow of patients through the study is shown in Fig. 1. The median number of therapy sessions received and the interquartile range (IQR) were 13.0 (IQR 8-23) for the CAT group and 11.0 (IQR 4.5-23) for the GCC group. The median numbers of individual non-therapy contacts (e.g. case management and psychiatrist appointments) were 33.0 (IQR 20.5-54.0) and 32.0 (IQR 18.5-52.5) respectively. There was no significant difference between the two groups on the number of therapy sessions or non-therapy contacts at any of the three follow-up time points (all $P>0.05$ ). The median interval from enrolment to discharge was 42.9 weeks (IQR 24.1-58.3) for the CAT group and 39.4 weeks (IQR 20.6-52.1) for the GCC group. The median number of contacts of any type per week was 1.4 (IQR 0.9-1.8) for the CAT group and 1.3 (IQR 0.8-1.6) for the GCC group.

Most patients who withdrew from the interventions continued to participate in research assessments (Fig. 1). Termination of therapy occurred with a formal final session (negotiated) or without a formal final session (drop-out). Negotiated termination was commonly because the patients believed that they had received enough treatment. There was no difference between the two study groups in the numbers of participants completing treatment, negotiating early termination or dropping out of treatment $\left(\chi^{2}=1.57, P=0.46\right)$.

Observed means and standard deviations at all four time points for the two groups are presented in Table 1 for the four continuous outcomes measures, whereas Table 2 contains the observed proportions in each category of parasuicidal behaviour. Summary statistics of the ten multiply imputed data-sets are available from the authors.

\section{Longitudinal treatment effects}

Both treatment groups demonstrated improvements over the 2-year period from baseline to final follow-up (based on the estimates displayed in the first row of Table 3). The GCC group evidenced a median improvement in absolute terms over all four continuous outcome measures of 0.88 standard deviation, whereas 


\begin{tabular}{|c|c|c|c|c|c|c|c|c|}
\hline \multirow[b]{2}{*}{ Outcome measure } & \multicolumn{2}{|c|}{ Baseline } & \multicolumn{2}{|c|}{6 months } & \multicolumn{2}{|c|}{12 months } & \multicolumn{2}{|c|}{24 months } \\
\hline & CAT $(n=41)$ & $\operatorname{GCC}(n=37)$ & CAT $(n=35)$ & $\operatorname{GCC}(n=34)$ & CAT $(n=36)$ & $\operatorname{GCC}(n=34)$ & CAT $(n=35)$ & $\operatorname{GCC}(n=33)$ \\
\hline BPD total score: mean (s.d.) & $19.27(3.13)$ & $19.14(3.67)$ & $18.46(3.15)$ & $19.21(3.70)$ & $17.97(3.61)$ & $18.38(4.13)$ & $17.06(4.62)$ & $16.76(5.01)$ \\
\hline SOFAS: mean (s.d.) & $60.27(8.40)$ & $61.16(10.53)$ & $67.31(9.81)$ & $65.06(11.35)$ & $67.36(11.64)$ & $67.71(11.70)$ & $71.74(11.56)$ & $75.30(12.21)$ \\
\hline Externalising: mean (s.d.) & $0.84(0.40)$ & $0.83(0.37)$ & $0.67(0.34)$ & $0.59(0.38)$ & $0.47(0.34)$ & $0.53(0.34)$ & $0.35(0.34)$ & $0.48(0.42)$ \\
\hline Internalising: mean (s.d.) & $1.03(0.35)$ & $0.97(0.40)$ & $0.75(0.51)$ & $0.65(0.41)$ & $0.60(0.50)$ & $0.59(0.42)$ & $0.48(0.44)$ & $0.52(0.48)$ \\
\hline
\end{tabular}

BPD, borderline personality disorder; CAT, cognitive analytic therapy; GCC, good clinical care; SOFAS, Social and Occupational Functioning Assessment Scale.

\begin{tabular}{|c|c|c|c|c|c|c|c|c|}
\hline \multirow[b]{2}{*}{ Frequency of parasuicide ${ }^{a}$} & \multicolumn{2}{|c|}{ Baseline } & \multicolumn{2}{|c|}{6 months } & \multicolumn{2}{|c|}{12 months } & \multicolumn{2}{|c|}{24 months } \\
\hline & CAT $(n=41)$ & GCC $(n=37)$ & CAT $(n=35)$ & GCC $(n=34)$ & CAT $(n=36)$ & GCC $(n=34)$ & CAT $(n=35)$ & $\operatorname{GCC}(n=33)$ \\
\hline None & 0.24 & 0.32 & 0.58 & 0.52 & 0.64 & 0.79 & 0.69 & 0.67 \\
\hline Monthly & 0.12 & 0.24 & 0.28 & 0.24 & 0.19 & 0.12 & 0.20 & 0.33 \\
\hline Weekly & 0.24 & 0.32 & 0.03 & 0.21 & 0.11 & 0.06 & 0.06 & 0.00 \\
\hline Daily & 0.12 & 0.24 & 0.11 & 0.03 & 0.07 & 0.04 & 0.06 & 0.00 \\
\hline
\end{tabular}

the median improvement for the CAT group was 1.02 standard deviations. Moreover, the two treatment groups showed a substantial reduction over time in the odds of a higher frequency of parasuicidal behaviour incidents $(\mathrm{OR}=0.32$ for $\mathrm{CAT}$ and $\mathrm{OR}=0.08$ for GCC).

\section{Differential rates of change}

Of critical interest was whether or not differences existed between the CAT and GCC groups in their relative rates of improvement from baseline to final follow-up. The second row of Table 3 shows that the expected rate of improvement was faster for CAT compared with GCC in externalising ( -0.50 s.d.) and internalising $(-0.29$ s.d.) pathologies, and moderately faster for GCC in general functioning $(-0.26$ s.d.); the upper boundary of the respective $95 \%$ confidence intervals (CIs), however, indicated these differential rates may at worst be slight. There was no meaningful or substantial difference between the two treatments in their respective rates of change over time for borderline personality disorder total scores and for frequency of parasuicidal behaviour.

\section{Group comparisons at final follow-up}

The third row of Table 3 reveals that the largest difference for cognitive analytic therapy over good clinical care at 24-month final follow-up was in externalising psychopathology ( -0.32 s.d.), with the upper limit of the $95 \%$ CI again indicating that this difference could also at worst be slight. No other noticeable difference at 24 months could be reasonably inferred on the remaining outcome measures.

\section{Discussion}

This is the first published randomised controlled trial of cognitive analytic therapy for any problem and it substantially enhances the evidence base for this psychotherapy. Two major findings emerge from this study. First, to our knowledge, this is the first study to show that early intervention for sub-syndromal or firstpresentation borderline personality disorder is possible and that it results in significant positive effects upon a broad range of patient outcomes. Second, patients in both treatment groups demonstrated significant and clinically substantial improvement, with cognitive analytic therapy showing some evidence of more rapid onset of benefit. These findings demonstrate 'proof of concept' for early intervention in borderline personality disorder and should also help to allay fears of iatrogenic harm arising from early diagnosis and treatment specifically for borderline personality disorder. ${ }^{31,32}$ The patients in this study were, on average, 13-15 years younger than those in recent randomised controlled trials

\begin{tabular}{|c|c|c|c|c|c|}
\hline & \multicolumn{5}{|c|}{ Estimate $(95 \% \mathrm{Cl})^{\mathrm{a}}$} \\
\hline & $\begin{array}{l}\text { Borderline personality } \\
\text { disorder total }^{\mathrm{b}}\end{array}$ & SOFAS ${ }^{b}$ & Internalising ${ }^{\mathrm{b}}$ & Externalising $^{\mathrm{b}}$ & Parasuicide $^{c}$ \\
\hline $\begin{array}{l}\text { Conditional rate of change for GCC } \\
\text { (baseline to } 24 \text { months) }^{\mathrm{d}}\end{array}$ & $-0.80(-1.22$ to -0.38$)$ & 1.19 (0.84 to 1.54$)$ & $-0.81(-1.13$ to -0.48$)$ & $-0.94(-1.34$ to -0.55$)$ & $0.08(0.02$ to 0.36$)$ \\
\hline $\begin{array}{l}\text { Group differential rate of change } \\
\text { for CAT } v . \text { GCC }^{e}\end{array}$ & $-0.09(-0.58$ to 0.40$)$ & $-0.26(-0.65$ to 0.13$)$ & $-0.29(-0.67$ to 0.10$)$ & $-0.50(-0.97$ to -0.04$)$ & 0.91 (0.17 to 4.98$)$ \\
\hline CAT v. GCC at 24 months $^{f}$ & $-0.10(-0.53$ to 0.32$)$ & $-0.21(-0.59$ to 0.18$)$ & $-0.11(-0.51$ to 0.28$)$ & $-0.32(-0.66$ to 0.02$)$ & 1.19 (0.27 to 5.24$)$ \\
\hline \multicolumn{6}{|c|}{$\begin{array}{l}\text { CAT, cognitive analytic therapy; GCC, good clinical care; SOFAS, Social and Occupational Functioning Assessment Scale. } \\
\text { a. Using } 10 \text { multiply imputed data-sets for the CAT group ( } n=41) \text { and GCC group ( }(n=37) \text {. } \\
\text { b. Partial standardised effects. } \\
\text { c. Conditional odds ratios. } \\
\text { d. Improvement over time indicated by (i) negative rate of change for borderline personality disorder total, internalising and externalising measures, (ii) positive rates of change for } \\
\text { SOFAS scores and (iii) odds ratios }<1 \text { for parasuicide. } \\
\text { e. Better improvement over time for CAT indicated by (i) negative differential rate of change for bordelrine personality disorder total, internalising and externalising measures, } \\
\text { (ii) positive rates of change for SOFAS scores and (iii) odds ratios }<1 \text { for parasuicide. } \\
\text { f. Better functioning at } 24 \text { months for CAT indicated by (i) negative standardised difference for borderline personality disorder total, internalising and externalising measures, } \\
\text { (ii) positive standardised difference for SOFAS scores and (iii) odds ratios }<1 \text { for parasuicide. }\end{array}$} \\
\hline
\end{tabular}


for borderline personality disorder. ${ }^{7,24,33,34}$ Moreover, their mean age was comparable to the mean age at first psychiatric contact reported in the randomised controlled trial by Clarkin et al (17 years) $)^{7,35}$ and was 5 years below that reported by Davidson et al (22 years). ${ }^{34}$ Only longer-term follow-up of this sample will demonstrate whether the gains made through early intervention are sustained and whether they divert patients from the poor outcomes associated with these traits and the course of disorder that leads to persistent problems and possibly to adult treatment settings.

Cognitive analytic therapy yielded the greatest median improvement on the four continuous outcome measures over the 2-year period. At 24 months, this therapy also showed a faster rate of improvement over time and lower levels of externalising psychopathology compared with good clinical care. However, the effect sizes for group differences in rate of change and at 24month follow-up are less than those hypothesised in the power analysis used for designing the study and represent medium-sized differences using Cohen's qualitative guidelines. ${ }^{36}$ Also, the effect of cognitive analytic therapy upon externalising psychopathology is interesting because the focus of this therapy is relational, not behavioural as in good clinical care. Further studies are required to examine possible mechanisms of action of this psychotherapy.

Both treatments were delivered exactly as they are intended to be delivered in clinical services and the HYPE clinic continues as a 'real world' service offering these interventions. The outcomes reported were achieved with a relatively small median 'dose' of therapy (13 CAT sessions and 11 GCC sessions) but delivered within a comprehensive service model. This included 2.5 case management sessions for every CAT session and 2.9 for every GCC session, highlighting that intervention involves more than just formal psychotherapy. This small dose of therapy does not necessarily imply that more would be better. The explicit time limit is an integral feature of cognitive analytic therapy, making it an exemplary intervention in terms of balancing the allocation of healthcare resources with the need for broad implementation of early intervention for borderline personality disorder. It also appears to be consistent with patient preferences and does not preclude future episodes of care.

The actual amounts of time spent with the CAT and GCC patients were almost identical, strengthening the claim that the findings favouring cognitive analytic therapy over good clinical care are actually due to CAT and that CAT adds value to the HYPE service model. The common elements of CAT and GCC (the HYPE service model) facilitate therapy delivery. They should not be viewed as incidental to treatment or as something to be controlled for in experimental designs, since they appear to be fundamental to the treatment models themselves.

Clinical experience suggests that 'treatment as usual' for borderline personality disorder ranges from untested specialised treatments through to mutually hostile clinical contact with likely iatrogenic harm ('maltreatment as usual'), ${ }^{31}$ and it is notable that GCC was not ineffective. This finding is consistent with the comparison by Linehan et al of 'treatment by experts' with historical treatment as usual. ${ }^{24}$ In contrast to treatment by experts, this study's good clinical care is a manualised, non-expert intervention that can be disseminated and replicated. It is possible that service reforms using existing resources and the HYPE model incorporating good clinical care might have important effects upon patient outcomes compared with the status quo of treatment as usual in most clinical services.

\section{Borderline personality disorder in adolescence}

Personality pathology is as important a form of psychopathology in adolescence as it is in adulthood, ${ }^{37}$ yet diagnosing adolescent personality pathology remains controversial. ${ }^{32}$ We have reviewed the growing body of evidence that indicates that a diagnosis of borderline personality disorder is no less reliable or valid in adolescence than it is in adulthood. ${ }^{4}$ It is widely acknowledged that personality disorders are best understood as dimensional constructs, ${ }^{38}$ and both sub-syndromal and full-syndrome borderline personality disorder in adolescence exist on a continuum of clinical severity and are prospectively associated with diverse functional and psychopathological poor outcomes, including a future diagnosis of borderline personality disorder, increased risk of Axis I disorders (especially substance use and mood disorders), interpersonal problems, distress and reduced quality of life. ${ }^{4}$ We do not claim that the patients in this study have the 'late-stage' borderline personality disorder syndrome described in DSM-IV and typically seen in adult mental health services. Like early intervention for first-episode psychosis, ${ }^{39}$ early intervention for borderline personality disorder usually involves including milder 'cases' or forms of disorder and targets the diverse outcomes associated with the presenting symptoms (e.g. borderline personality disorder, Axis I pathology, interpersonal problems) rather than narrowly focusing upon the 'late-stage' syndrome, ${ }^{4,39}$ especially as progression to symptomatically chronic borderline personality disorder is uncommon, even in adulthood. ${ }^{40}$

\section{Strengths of the study}

Good clinical care was an active, non-specialised intervention that was clearly characterised, with documented good adherence and equal access to the elements of care not included in formal psychotherapy. This provided a more rigorous control condition than the common comparison condition of treatment as usual, which is seldom characterised. Also, both cognitive analytic therapy and good clinical care were delivered in their intended 'real world' form and with equal access to a comprehensive service model of care, which was characterised and measured. Also, the study was conducted in a front-line clinical service, with clinical referrals from the community and with few exclusion criteria, enhancing its external validity.

Using the same therapists to deliver both treatment conditions allowed for rigorous control of some therapist effects (gender, experience, personality). However, it also allowed for possible treatment 'contamination' or sabotage. The GCC treatment integrity measure and random peer audits revealed excellent adherence to the model and negligible overt contamination. However, covert contamination was still possible, i.e. 'thinking in CAT'. Additionally, although our group were not cognitive analytic therapy 'insiders', arguably reducing therapist allegiance effects, the team reported developing a preference for this form of therapy during the trial. Also, the therapists were experienced in cognitive-behavioural therapy but new to cognitive analytic therapy. Treatment effects might have been more pronounced with greater experience in the latter therapy.

\section{Limitations}

The sample size, although comparable with other published randomised controlled trials for adult borderline personality disorder, is still relatively small in absolute terms and restricts the degree of precision for detecting the small to medium kinds of treatment effects found in our study. Also, participants were not randomised to therapists, allowing some potential biasing through matching of patient to therapist. It would also have been preferable to perform the randomisation procedure after baseline assessment. We cannot say whether the eight participants who withdrew prior to baseline assessment did so as a result of their 
assignment to a particular treatment group. Finally, as this is the first study of its kind, the findings reported are preliminary and need replication.

\section{Implications of the study}

This study should improve clinicians' confidence that a diagnosis of sub-syndromal or full-syndrome adolescent borderline personality disorder can now be matched with an effective intervention. Cognitive analytic therapy might be preferred to good clinical care, but the latter was not ineffective and service reform using this model might be achieved more swiftly, with cognitive analytic therapy implementation as a second phase of development. Further studies are required to replicate these findings and to investigate the mechanisms of change, the sustainability of the improvements beyond 2 years and the effectiveness of cognitive analytic therapy in samples of older people with borderline personality disorder.

\section{Acknowledgements}

The authors thank all participants in this study and the staff of ORYGEN Youth Health. Particular thanks go to Dr Anthony Ryle, Dr lan Kerr, Ms Eva Burns-Lundgren, Dr Dawn Bennett, Dr Jackie Withers and the Association for Cognitive Analytic Therapy (UK) for training and supervision in cognitive analytic therapy. Thanks also go to Associate Professor John Gleeson for supervision of standardised good clinical care, Dr Andrew Court for independent psychiatric assessments, Dr Carol Hulbert, Ms Helen Mildred and Dr Denise Charman for advice on the implementation of the study and to Professor Anthony Jorm and Dr Sarah Hetrick for comments on an earlier version of this manuscript. This work was supported by grants $98-0198$ from the Victorian Health Promotion Foundation, Melbourne, Australia and grant 990748 from the National Health and Medical Research Council Canberra, Australia. The ORYGEN Research Centre is supported by an unrestricted grant from the Colonial Foundation, Melbourne, Australia.

Andrew M. Chanen, MBBS, MPM, FRANZCP, ORYGEN Research Centre, Department of Psychiatry, University of Melbourne, and ORYGEN Youth Health, Northwestern Mental Health, Melbourne; Henry J. Jackson, PhD, FAPS, School of Behavioural Science, University of Melbourne; Louise $\mathbf{K}$. Mccutcheon, Dpsych, ORYGEN Research Centre, Department of Psychiatry, University of Melbourne, and ORYGEN Youth Health, Northwestern Mental Health, Melbourne; Martina Jovev, PhD, Youth Health, Northwestern Mental Health, Melbourne; Martina Jovev, PhD,
ORYGEN Research Centre, Department of Psychiatry, University of Melbourne; Paul Dudgeon, PhD, School of Behavioural Science, University of Melbourne; Hok Pan Yuen, MSC, ORYGEN Research Centre, Department of Psychiatry, University of Melbourne; Dominic Germano, Mpsych, Helen Nistico, Mpsych ORYGEN Youth Health, Northwestern Mental Health, Melbourne; Emma McDougall, BSc, Caroline Weinstein, BSC, Verity Clarkson, BSC, ORYGEN Research Centre, Department of Psychiatry, University of Melbourne; Patrick D. McGorry, MD, PhD FRANZCP, ORYGEN Research Centre, Department of Psychiatry, University of Melbourne, and ORYGEN Youth Health, Northwestern Mental Health, Melbourne, Australia

Correspondence: Dr Andrew Chanen, ORYGEN Research Centre, Locked Bag 10, Parkville, Victoria 3052, Australia. Email: achanen@unimelb.edu.au

First received 14 Dec 2007, final revision 15 Jul 2008, accepted 30 Jul 2008

\section{References}

1 American Psychiatric Association. Diagnostic and Statistical Manual of Mental Disorders (4th edn, Text Revision) (DSM-IV-TR). APA, 2000.

2 Chanen AM, Jovev M, Jackson HJ. Adaptive functioning and psychiatric symptoms in adolescents with borderline personality disorder. J Clin Psychiatry 2007; 68: 297-306.

3 Zanarini MC, Frankenburg FR, Khera GS, Bleichmar J. Treatment histories of borderline inpatients. Compr Psychiatry 2001; 42: 144-50.

4 Chanen AM, Jovev M, McCutcheon L, Jackson HJ, McGorry PD. Borderline personality disorder in young people and the prospects for prevention and early intervention. Curr Psychiatry Rev 2008; 4: 48-57.

5 Mrazek PJ, Haggerty RJ. Reducing Risks for Mental Disorders: Frontiers for Preventive Intervention Research. National Academy Press, 1994.

6 Eaton WW, Badawi M, Melton B. Prodromes and precursors: epidemiologic data for primary prevention of disorders with slow onset. Am J Psychiatry 1995; 152: 967-72.

7 Clarkin JF, Levy KN, Lenzenweger MF, Kernberg OF. Evaluating three treatments for borderline personality disorder: a multiwave study. Am J Psychiatry 2007; 164: 922-8.
8 Ryle A. Cognitive Analytic Therapy of Borderline Personality Disorder. The Model and the Method. Wiley, 1997.

9 Linehan MM. Behavioral treatments of suicidal behaviors. Definitional obfuscation and treatment outcomes. Ann N Y Acad Sci 1997; 836: 302-28.

10 Cohen P. Childhood risks for young adult symptoms of personality disorder: method and substance. Multivariate Behav Res1996; 31: 121-48.

11 Johnson JG, Cohen P, Brown J, Smailes E, Bernstein DP. Childhood maltreatment increases risk for personality disorders during early adulthood. Arch Gen Psychiatry 1999; 56: 600-6.

12 McGorry PD, Parker AG, Purcell R. Youth mental health: a new stream of mental health care for adolescents and young adults. In Mental Health in Australia: Collaborative Community Practice (2nd edn) (eds G Meadows, B Singh \& M Grigg): 438-49. Cambridge University Press, 2007.

13 First MB, Gibbon M, Spitzer RL, Williams JBW. Structured Clinical Interview for DSM-IV Axis I Disorders-Patient Version (SCID-I/P). Biometrics Research Department, New York State Psychiatric Institute, 1996.

14 Kaufman J, Birmaher B, Brent D, Rao U, Flynn C, Moreci P, Williamson D, Ryan N. Schedule for Affective Disorders and Schizophrenia for School-Age Children-Present and Lifetime version (K-SADS-PL): initial reliability and validity data. J Am Acad Child Adolesc Psychiatry 1997; 36: 980-8.

15 First MB, Gibbon M, Spitzer RL, Williams JBW, Benjamin LS. User's Guide for the Structured Clinical Interview for DSM-IV Axis II Personality Disorders. American Psychiatric Press, 1997.

16 Pilkonis PA, Heape CL, Ruddy J, Serrao P. Validity in the diagnosis of personality disorders: the use of the LEAD standard. Psychol Assess 1991; 3: 46-54.

17 Bernstein DP, Cohen P, Skodol A, Bezirganian S, Brook J. Childhood antecedents of adolescent personality disorders. Am J Psychiatry 1996; 153: 907-13.

18 Achenbach TM. Manual for the Youth Self-Report and 1991 Profiles. Department of Psychiatry, University of Vermont, 1991.

19 Ivanova MY, Achenbach TM, Rescorla LA, Dumenci L, Almqvist F, Bilenberg N, Bird $\mathrm{H}$, Broberg AG, Dobrean A, Dopfner M, Erol N, Forns M, Hannesdottir $\mathrm{H}$, Kanbayashi $Y$, Lambert MC, Leung $P$, Minaei A, Mulatu MS, Novik T, Oh KJ, Roussos A, Sawyer M, Simsek Z, Steinhausen HC, Weintraub S, Winkler Metzke C, Wolanczyk T, Zilber N, Zukauskiene R, Verhulst FC. The generalizability of the Youth Self-Report syndrome structure in 23 societies. J Consult Clin Psychol 2007; 75: 729-38.

20 Achenbach TM. Manual for the Young Adult Self-Report and Young Adult Behavior Checklist. Department of Psychiatry, University of Vermont, 1997.

21 Goldman H, Skodol A, Lave T. Revising Axis V for DSM-IV: a review of measures of social functioning. Am J Psychiatry 1992; 149: 1148-56.

22 Ryle A, Kerr IB. Introducing Cognitive Analytic Therapy. Wiley, 2002.

23 Ryle A. The contribution of cognitive analytic therapy to the treatment of borderline personality disorder. J Personal Disord 2004; 18: 3-35.

24 Linehan MM, Comtois KA, Murray AM, Brown MZ, Gallop RJ, Heard HL, Korslund KE, Tutek DA, Reynolds SK, Lindenboim N. Two-year randomized controlled trial and follow-up of dialectical behavior therapy vs therapy by experts for suicidal behaviors and borderline personality disorder. Arch Gen Psychiatry 2006; 63: 757-66.

25 Treatment Protocol Project. Management of Mental Disorders (2nd edn). World Health Organisation Collaborating Centre for Mental Health and Substance Abuse, 1997.

26 Cohen J. Statistical Power Analysis for the Behavioral Sciences (2nd edn). Erlbaum, 1988.

27 Schafer JL, Graham JW. Missing data: our view of the state of the art. Psychol Methods 2002; 7: 147-77.

28 Schafer JL. Multiple imputation with PAN. In New Methods for the Analysis of Change (eds AG Sayer \& LM Collins): 355-77. American Psychological Association, 2001.

29 R Development Core Team. R: A Language and Environment for Statistical Computing. 2.5.1. R Foundation for Statistical Computing; 2007.

30. Rubin DB. Multiple Imputation for Nonresponse in Surveys. Wiley, 1987.

31 Fonagy $\mathrm{P}$, Bateman A. Progress in the treatment of borderline personality disorder. Br J Psychiatry 2006; 188: 1-3.

32 Chanen AM, McCutcheon LK. Personality disorder in adolescence: the diagnosis that dare not speak its name. Personal Ment Health 2008; 2 35-41.

33 Giesen-Bloo J, van Dyck R, Spinhoven P, van Tilburg W, Dirksen C, van Asselt T, Kremers I, Nadort M, Arntz A. Outpatient psychotherapy for borderline personality disorder: randomized trial of schema-focused therapy vs transference-focused psychotherapy. Arch Gen Psychiatry 2006; 63: $649-58$ 
34 Davidson K, Tyrer P, Gumley A, Tata P, Norrie J, Palmer S, Millar H, Drummond L, Seivewright $H$, Murray $H$, Macaulay F. A randomized controlled trial of cognitive behavior therapy for borderline personality disorder: rationale for trial, method, and description of sample. J Personal Disord 2006; 20: $431-49$.

35 Clarkin JF, Levy KN, Lenzenweger MF, Kernberg OF. The Personality Disorders Institute/Borderline Personality Disorder Research Foundation randomized control trial for borderline personality disorder: rationale, methods, and patient characteristics. J Personal Disord 2004; 18: 52-72.

36 Cohen J. A power primer. Psychol Bull 1992; 112: 155-9.
37 Westen $D$, Dutra L, Shedler J. Assessing adolescent personality pathology. Br J Psychiatry 2005; 186: 227-38.

38 Clark LA. Assessment and diagnosis of personality disorder: perennial issues and an emerging reconceptualization. Annu Rev Psychol 2007; 58: 227-57.

39 McGorry PD. Issues for DSM-V: clinical staging: a heuristic pathway to valid nosology and safer, more effective treatment in psychiatry. Am J Psychiatry 2007; 164: 859-60.

40 Zanarini MC, Frankenburg FR, Hennen J, Reich DB, Silk KR. Prediction of the 10-year course of borderline personality disorder. Am J Psychiatry 2006; 163 827-32.

\section{Mark Rothko (1903-1970)}

Text by Alexandra Pitman

Mark Rothko's darker works began in 1957 as he entered the final decade of his life. The current Rothko exhibition at Tate Modern in London focuses on five series of works produced between 1958 and 1970, the most significant being a commission for the Four Seasons Restaurant in Manhattan's Seagram Building. In this exhibition the Seagram murals are hung in a cathedral-like space, in keeping with the artist's stringent specifications over positioning and environment. These images, most notably Red on Maroon Mural Section 4 (1959), exemplify his signature brooding multiforms and their ability to convey atmospheres. Rothko became unhappy about the prospect of his murals hanging in a buzzing restaurant where there was little scope for any participation with them. He dismissed the idea as mere interior design for the rich and decided to pull out of the commission, later donating a selection to the Tate to be viewed in a dedicated Rothko Room. The paintings arrived in London on the day of his suicide in February 1970.

The panels of colour and ragged borders so recognisable in these works are grimly echoed in his death. Rothko's body was found on the floor of his studio the morning after his overdose, having also severed his brachial arteries. Around his body blood had formed a rectangular pool approximately the size of one of his canvases. In his final years the Black-Form paintings (1964), the Brown on Grey works (1969) and the Black on Gray series (1969-70) expressed his failing health and hopes. Drinking and smoking heavily and afflicted by gout, shingles, liver cirrhosis, bronchitis and another failed marriage, Rothko's work showed an increasing prominence of what he described as 'the tragic ingredient'. Although there is a tendency to turgid analysis of Rothko's work there is one thing on which most agree: that the paintings of his last 2 years reflect an intensifying preoccupation with dissolution and with death

Rothko is at Tate Modern, Bankside, London SE1 9TG, UK until 1 February 2009

http://www.tate.org.uk/modern/exhibitions/markrothko/ 